\title{
Correct a Wide Spread Conclusion of Cantor Set Theory
}

\author{
Ming Xiong \\ Independent Scholar, Zhongxian, Chongqing, China \\ Email: x599599@126.com
}

Received 10 May 2015; accepted 5 July 2015; published 8 July 2015

Copyright (C) 2015 by author and Scientific Research Publishing Inc.

This work is licensed under the Creative Commons Attribution International License (CC BY). http://creativecommons.org/licenses/by/4.0/

\section{(c) (i) Open Access}

\begin{abstract}
The Cantor's conclusion that "there is a one-one correspondence between the points on n-dimensional space and the points on the line" appears in the numerous current documents. By introducing a monotonic and continuous function, a one-one correspondence between two intervals is built; and by using parametric equations of the curve, a one-one correspondence from the points on the curve to the points on the line is established. Specially, the meanings of multivariate functions are given. By using a n-variable equation with a parameter, a correspondence from n-dimensional space area to a interval is built, so the wrong conclusion is completely denied. The paper enriches calculus and can reduce the teaching difficulty of real function in some degree. The expression of moving curve (surface) limit is given in the paper. More importantly, after the conclusion is corrected, it will be necessary and possible to re-establish the theory and the approach about multivariable differential calculus.
\end{abstract}

\section{Keywords}

Cantor, Error of Set Theory, Correspondence, Meanings of Multivariate Function

\section{Introduction}

Cantor was certainly one of the great mathematicians. Based on the study results of Dirichlet and Riemann, he developed point-set theory, and exploited a new world—set theory. Because his creative work clashed with mathematical traditional view, he encountered the strong opposition from academic authorities such as Klein, Weyl, Poincare. But he received strong support from other authorities such as Dedekind, Weierstrass, Mittag-Leffler. Hilbert spok highly of Cantor Set Theory and rated continuum hypothesis the top of the famous 23 probems at the 2nd ICM in 1900. Set theory had been generally accepted by us in the 20th century. Although Russells, a logician, praised of Cantor too, he found Cantor' set theory was not complete, and he proposed a paradox in 1901:

Suppose $R=\{x \mid x \notin x\}$, then the necessary and sufficient condition of $R \notin R$ is $R \in R$. 
The accessible version of the paradox is "barber paradox" or "liar paradox", which results in the 3th mathematics crisis. At present, set theory has gone deep among many branches of mathamatics, and can describe mathematical concepts or theorems precisely and rigorously, but we detect that there is still the other flaw in Cantor set theory.

\section{A Wide Spread Conclusion of Cantor Set Theory}

In 1874, Cantor started to consider whether there is a one-one correspondence between the points on the line and the points in the n-dimensional space. After 3 years' hard work, he said proudly that "there is a one-one correspondence between n-dimensional space and a line, and the points on n-dimensional space are as many as on a line" in his private communication with Dedekind. Since Cantor set theory had been published, mathematicians all over the world have taken the conclusion for granted. To this day, hardly anyone questions the conclusion, it has appeared in numerous textbooks of high school maths [1] 75, mathematical history [2] 227, and the equivalent proposition of the conclusion:

Suppose the sign $C$ denotes the cardinality of continuum (an interval or the line), then the cardinality of $E^{n}$ or $E^{\infty}$ (n-dimensional space or infinite dimensional space) is still $C$. has appeared in numerous textbooks of set theory and real variable function [3] [4], etc.

\section{The Process of Discovering the Wrong Conclusion}

In the process of studying further multivariable calculus, it was not until the end of 2013 year that I discovered the conclusion to be obviously wrong. I had also taken the conclusion for granted when I studied real variable function and mathematical history 25 years ago. In 2006, I started to teach higher mathematics and took up with element method and tried to solve multiple integral with element method. Based on the results of Tang Yanzeng and others [5], we proposed concepts of moving curve (surface), by which multiple integrals, except surface integral of the second type, can be reduced to simple integrals theoretically. But, before this, scholastic circles seem to believe that only special multiple integrals can be reduced to simple integrals. In the process of further researching conditional extremum, we discovered many new meanings of multivariate function which fully indicates that the conclusion of Cantor is wrong.

In order to lead more broader people to realize the wrong conclusion, and in order to dispel the myths that it created and to remove barriers to studying in mathematics, we will use the concept of mapping, moving curve (surface), meanings of multivariate function to reveal and correct the wrong conclusion.

\section{One-One Correspondence}

The origin, per unit length and positive direction are determined on a line, every real number corresponds to the only point on number axis, every spot on number axis corresponds to the only real number. Under rectangular coordinate system, each point in the plane corresponds to only the ordered pair (a, b), and each ordered pair (a, b) corresponds the only point in the plane. The class of correspondences is called one-one correspondences.

\subsection{The Definition of One-One Correspondence}

Mapping: Let $A$ and $B$ be two sets. According to certain corresponding rule $f$, if each element $a$ in $A$ has the only element $b$ in $B$, the rule $f$ is said to be mapping from $A$ to $B$, which is expressed $f: A \rightarrow B . a$ in $A$ is called $a$ preimage, and $b$ in $B$ is called an image.

One-one correspondence: Let $f: A \rightarrow B$ be a mapping from $A$ to $B$. If each element $b$ in $B$ has the only preimage $a$, the mapping $f: A \rightarrow B$ is called one-one correspondence (or one-one mapping, one-one transformation).

Any function of one variable is a mapping from domain to value region, where an independent is a preimage and a function value is an image. Monotone functions are one-one correspondences. Quadratic functions are two-one mappings. Periodic functions are "infinitely many-one" mappings.

\subsection{One-One Correspondence from an Interval to the Other Interval}

Using $y=\frac{12}{5} x$, Bolzano (Czekh, 1781-1849) constructed one-one correspondence from the interval $[0,5]$ to 
the interval $[0,12]$. He proposed that there were the same many in set $\mathrm{A}$ as that in set $\mathrm{B}$, if a one-one correspondence existed in between A and B.

Actually, when the two intervals are regard as the domain and the value region separately, using a appropriate monotone and continuous function, the one-one relationship is established.

Example 1 Establish one-one correspondences from an interval to the other interval.

1) $[a, b) \rightarrow[c, d)$,

2) $(-\infty,+\infty) \rightarrow(0,1)$,

3) $(0,1] \rightarrow[0,+\infty)$,

4) $[a, b] \rightarrow(-\infty,+\infty),[a, b]$ is any interval.

Solution: (1) $[a, b)$ and $[c, d)$ are regard as the domain and the value region separately, then the one-one correspondence is established by $y=\frac{d-b}{c-a}(x-a)+b$.

2) $y=\pi^{-1} \operatorname{arccot} x, x \in R, y \in(0,1)$.

3) $y=\log _{0.5} x, x \in(0,1], y \in[0,+\infty)$.

4) Firstly, we determine the altitude points $\mathrm{A}$ and $\mathrm{B}$ right above $a, b$ on number axis and drow a smooth and concave curve $\mathrm{L}$ between $\mathrm{A}$ and $\mathrm{B}$. Secondly, we draw a ray line from the midpoint $\mathrm{P}$ of $\mathrm{AB}$ to intersect $\mathrm{L}$ and the number axis at $\mathrm{M}$ and $\mathrm{N}$ separately. Thus $\mathrm{M}$ corresponds to $\mathrm{N}$, and the horizontal coordinate of $\mathrm{M}$ corresponds to $\mathrm{N}$ on number axis. So, the correspondence is established between $[a, b]$ and $(-\infty,+\infty)$.

Strictly speaking, there is no one-one correspondence between open-interval and closed interval unless the two endpoints on closed interval are removed, and it is as well between open interval and half-open interval or between closed interval and half-open interval.

As these examples illustrate: there are as many real numbers on $\mathrm{R}$ as on any open interval of $\mathrm{R}$, as well as between any interval and the line.

\subsection{One-One Correspondence between a Curve and the Line}

Every point $(x, f(x))$ on the graph of a function $y=f(x)$ corresponds the only point $x$ on the domain. If the function is continuous, the graph of it is a continuous curve. For any given curve, by using geometrical method or algebraic means, the one-one relation is established between the points on the curve and on the line, which can show that there are the same points on the curve as on the line.

Example 2 [6] The points on a circle are the same many as the points on the line.

Proof: Method-1. Let a circle touch the number axis at the origin (the tangent point corresponds to the origin). Let a tangent line from any point $\mathrm{A}$ on the circle intersect the number axis at a, then point $\mathrm{A}$ on the circle corresponds to a on the line (Set the tangent point corresponds to $-\infty$ or $+\infty$, when the tangent line and the number axis are parallel to each other), and the points on the circle are the same many as on the line. Because any line segment can be formed a circle and the points on any circle are the same many as on the number axis ,the pints on a circle or on any line segment are the same many as on the line.

Method-2 The function $u=2 \pi-2 \operatorname{arccot} t$ establishes the one-one correspondence from $(-\infty,+\infty)$ to $(0,2 \pi)$, and the parameter equations

$$
\left\{\begin{array}{l}
x=\frac{R}{\sqrt{6}} \sin u+\frac{R}{\sqrt{2}} \cos u \\
y=\frac{R}{\sqrt{6}} \sin u-\frac{R}{\sqrt{2}} \cos u \\
z=-\frac{2 R}{\sqrt{6}} \sin u
\end{array}\right.
$$

establishes the one-one correspondence from interval $(0,2 \pi)$ to the circle 


$$
\left\{\begin{array}{l}
x^{2}+y^{2}+z^{2}=R^{2} \\
x+y+z=0
\end{array} .\right.
$$

So

$$
\left\{\begin{array}{l}
x=-\frac{R}{\sqrt{6}} \sin (2 \operatorname{arccot} t)+\frac{R}{\sqrt{2}} \cos (2 \operatorname{arccot} t) \\
y=-\frac{R}{\sqrt{6}} \sin (2 \operatorname{arccot} t)-\frac{R}{\sqrt{2}} \cos (2 \operatorname{arccot} t) \\
z=\frac{2 R}{\sqrt{6}} \sin (2 \operatorname{arccot} t)
\end{array}\right.
$$

establishes the one-one correspondence from $(-\infty,+\infty)$ to circle. Note: The point $\left(\frac{R}{\sqrt{2}},-\frac{R}{\sqrt{2}}, 0\right)$ corresponds to $-\infty$ or $+\infty$.

\section{Countable Set and Uncountable Set}

Cantor proposed that the set was called countable if the all elements in the set could be arranged at certain regular. He divided all the sets into two types: countable sets and uncountable sets, and proposed that the set of integers and the set of rational numbers were all countable, and there were as many positive rational numbers as positive integers. The set of irrational number and the set of real number are all uncountable. In spite of infinitely many rational numbers and real numbers between any two real number, each rational number can be expressed as the quotient of two integers [7], thus rational numbers can be arranged at a certain manner. But, any irrational number is a non-repeating and non-terminating decimal number, and anyhow cannot be expressed as the quotient of two integers, it can only be the arithmetic of adding, subtracting, multiplying, and dividing for infinitely many integer numbers, or it can only be the limit of a sequence of rational numbers. Irrational numbers on $(-\infty,+\infty)$ can not be arranged through any means. The above all are the differences and relations.

Example 3 There are the same rational numbers on any interval as positive integers.

Proof For convenience, we will first prove that the number of rational numbers on interval $(0.1,0.2)$ is equal to number of positive integers.

We arrange the all rational numbers on the interval under the rules: ascending denominator (if the denominator is the same, the rule is ascending numerator; if a rational number is equal to the former, keep the former). So, the all rational numbers on the interval $(0,1,0,2)$ is arranged as follows.

$\frac{2}{11}, \frac{2}{12}, \frac{2}{13}, \frac{2}{14}, \frac{2}{15}, \frac{2}{16}, \frac{3}{16}, \frac{2}{17}, \frac{3}{17}, \frac{2}{18}, \frac{2}{19}, \frac{3}{19}, \frac{3}{20}, \frac{3}{21}, \frac{4}{21}, \frac{3}{22}, \frac{3}{23}, \frac{4}{23}, \cdots$ As the denominators are increasing and tending to infinity, the all rational numbers on $(0.1,0.2)$ are lined up as a sequence. Each rational number on the interval corresponds to the only one positive integer. The all rational number on any interval are similarly arranged in the above maner.

The number of elements in a set is called the cardinal number or cardinality of the set. Any interval is a continuum, $C$ is used to express the cardinality of any interval [3].

\section{The Mapping from n-Dimensional Space to Interval}

The system of coordinates has built a bridge between algebra and geometry. Any binary equation with infinitely many solutions represents a curve (line, half-line, polyline), for instance $y=|x|$ denotes a V-type curve. Any function of one variable is binary equation and denotes a curve. Similarly, any function of two variables is a ternary equation and denotes a surface, for instance $z=x^{2}+y^{2}$ denotes a paraboloid of revolution. In the process of changing multiple integrals or surface integrals directly into simple integrals, an equation with a parameter [8]-[11]

$$
F\left(x_{1}, x_{2}, \cdots, x_{n}\right)=t
$$

is introduced, which is called to be a degenerate transformation. $F\left(x_{1}, x_{2}, \cdots, x_{n}\right)=t$ expresses a moving sur- 
face (when $\mathrm{n}=2$, it expresses a moving curve), and is used to describe or simulate continuous variation of object. But, it has many significances.

\subsection{The Mapping from Planar Domain to Interval}

Example 4 Give the meanings of the equations

$$
\begin{aligned}
& \text { 1) } y=t x^{2} \\
& \text { 2) } x y=t(t>0)
\end{aligned}
$$

Solution: (1) When $t$ is assigned a non-zero real number, a parabola is obtained from the Equation (2). When $t=0$ and $y=0$, (2) expresses $x$-axis. When $t$ has travered all the real numbers, a family of parabola in the plane is obtained, which have a common apex and a common axis of symmetry. Each parabola and all points on it correspond to the only real number, and vice versa. So, Equation (2) establishes a correspondence from the coordinate plane to the interval $(-\infty,+\infty)$. When $t$ is continuously changing on $(-\infty,+\infty)$, (2) represents a moving parabola. The smaller $|t|$ is, the large the opening of the moving parabola is. When $t \rightarrow 0$, the moving parabola is infinitely tending to $\mathrm{x}$-axis; when $t \rightarrow \infty$, the moving parabola is tending to y-axis. Using symbols of set and limit, the two ultimate states are expressed as respectively:

$$
\begin{aligned}
& \lim _{t \rightarrow \infty}\left\{l: y=t x^{2}, t \in R\right\}=\left\{l_{1}: x=0\right\}, \\
& \lim _{t \rightarrow 0^{+}}\left\{l: y=t x^{2}, t \in R\right\}=\left\{l_{2}: y=0\right\} .
\end{aligned}
$$

(2) The Equation (3) $x y=t(t>0)$ denotes a family of hyperbola in the first and the third quadrants. They have common symmetry axis (line $y=x$ ) and a common asymptote (the $\mathrm{x}$ and the $\mathrm{y}$ coordinate axes), the vertex of any hyperbola is on the line $y=x$. Each hyperbola and the points on it are correspond to the only real number, and vice versa. (3) establishes a mapping from the first quadrant and the third quadrant to the interval ( 0 , $+\infty)$.

When $t \rightarrow+\infty$, the moving hyperbola is moving to infinity along the line $y=x$. But, when $t \rightarrow 0^{+}$, the moving hyperbola is tending to the two axis, which is the limit state of the moving curve and is expressed as

$$
\lim _{t \rightarrow 0^{+}}\{l: x y=t, t>0\}=\left\{l_{0}: \text { the two axis }\right\} .
$$

So, the equation $F(x, y)=t$ expresses a moving curve and a family of curves in the plane, and a mapping.

\subsection{The Mapping from n-Dimensional Domain to Interval}

Analogously, any equation $F(x, y, z)=t$ has three meanings: a family of surfaces, a moving surface and a mapping.

Example 5 Give the meanings of the equations

1) $x+y+z=t(t \in R)$

2) $x^{2}+y^{2}+z^{2}=t(t>0)$

3) $[8] \quad x y z=t$

\section{Solution:}

1) (4) acts as a moving plane and a family of planes in the space; a mapping that makes each plane and the infinite points in the each plane correspond to the only real number, and vice versa.

2) (5) acts as a moving sphere and a family of spheres in the space; a mapping that makes each sphere and the infinite points in each sphere correspond to the only positive number, and vice versa.

3 ) When $t$ is assigned a non-zero real number, a four-leaf surface is obtained from the equation; when $t$ is assigned a positive real number, the four-leaf surface is located $1,3,6,8$ four octants; when $t$ is assigned a minus, the four-leaf surface is located 2, 4, 5, 7 four octants.

For convenience, the case in 1 octant is discussed. When $t$ has gone through real numbers on $(0,+\infty)$, $x y z=t(x, y, z, t>0)$ expresses a family of surfaces, which have a common asymptotic surface (three coordi- 
nate planes in 1 octant), all the vertex $(\sqrt[3]{t}, \sqrt[3]{t}, \sqrt[3]{t})$ on the line $x=y=z$.

The equation $x y z=t(x, y, z, t>0)$ establishes a mapping from 1 octant to the $(0,+\infty)$, which make each surface and the infinite points in the each surface correspond to the only positive number.

When $t \rightarrow+\infty$, the moving surface is moving tending towards infinity along the line $x=y=z$. When $t \rightarrow 0^{+}$, it is tending to the three coordinate planes in the 1 octant, which is the limit state of the moving surface. It is expressed as $\lim _{t \rightarrow 0^{+}}\{\Sigma: x y z=t, x, y, t>0\}=\left\{\Sigma_{0}\right.$ : three coordinate planes in the loctant $\}$.

\section{The Meanings of Multivariate Functions}

An equation $F(x, y)=t$ is actually a function of two variables $z=F(x, y)$. Thus, the meanings of a function of two variables $z=F(x, y)$ are as follow: a surface in space, a moving curve; a family of curves; a mapping from the domain to the value region of the function, which makes each curve and all the points on the each curve correspond to the only value of the function.

Functions of a variable can be classified into two kinds: one-one correspondence, several-one correspondence. But, any function of two variables is a infinity-one correspondence (the extreme points and the points of maxima or minima can are excepted).

Choose any function value $z_{0}$ (non-extremum and non-maximum, non-minimum), then $F(x, y)=z_{0}$ is a planar curve, on which the infinite points are corresponding to the only $z_{0}$.

Owing to infinitely many real numbers between any two real numbers, there are infinitely many curves between any two curves

$$
F(x, y)=z_{1}, F(x, y)=z_{2}
$$

so the domain of the function $z=F(x, y)$ is completely covered with a family of curves $F(x, y)=t$.

Similarly, $F(x, y, z)=t$ is a function of three variables $u=F(x, y, z)$. Thus, the meanings of a function of three variables $u=F(x, y, z)$ are as follows: a moving surface and a family of surfaces in the space; a mapping from the domain to the value region of the function, which makes each surface and all the points in the each surface correspond to the only value of the function. The domain of the function $u=F(x, y, z)$ is completely filled with a family of surfaces.

Any function of three variables is also an infinite many-one correspondence (the extreme points, the points of maxima or minima are excepted).

A function value $u_{0}$ (non-extremum and non-maximum, non-minimum) is given, then $F(x, y, z)=u_{0}$ is a surface in which the infinite points correspond to the only $u_{0}$.

Generally, a continuous function of two variables expresses a surface in the space; a degenerate transformation; a moving curve in the area; a family of curves that cove completely the whole area; a mapping from the area to the value region of the function, which makes each curve and all the points on the each curve correspond to the only value of the function. Any continuous function of $n$-variables $(n \geq 3)$ expresses a moving surface in $\mathrm{n}$-dimensional space; a degenerate transformation; a family of surfaces that fill completely in the $\mathrm{n}$-dimensional domain; a mapping from the n-dimensional domain to the value range of the function, which makes each surface and all the points on the each surface correspond to the only value of the function. Any multivariate function is a infinite many-one correspondence from the points of the domain to the points of the functional value.

Furthermore, because any binary equation with infinite many solutions expresses a curve, and a curve is a one-dimensional space, so the binary equation expresses a one-dimensional space, and a function $z=F(x, y)$ expresses a moving one-dimensional space and a family of one-dimensional spaces in the domain of the function. Similarly, any ternary equation with infinite many solutions expresses a surface, and a surface is a twodimensional space, so the ternary equation expresses a two dimensional space, and a function $u=F(x, y, z)$ expresses a moving two dimensional subspace and a family of two dimensional subspaces in the domain of the function. By extension, a function $z=F\left(x_{1}, x_{2}, \cdots, x_{n}\right)(n \geq 2)$ expresses a moving $n-1$ dimensional subspace and a family of $n-1$ dimensional subspaces in the domain of the function, and the family of $n-1$ dimensional subspaces correspond to the points on the rang of the function of $n$ variables. The cardinality of a family $n-1$ dimensional subspaces in n-dimensional space is equals to the cardinality of any interval. 


\section{Correction for Cantor' Conclusion and Significances}

For any n-dimensional domain and any interval, let the domain be the domain of a function of $\mathrm{n}$ variables and the interval be the rang of the function, a one-one correspondence from a family of $n-1$ dimensional subspace to the interval is established by the function. As long as a one-one correspondence is established between set $\mathrm{A}$ and set B, the elements in A are the same many as that in B. Due to the one-one correspondence from any interval to the line, and owing to the one-one correspondence from a family of $n-1$ dimensional spaces in $n$-dimensional space to an interval, there is a one-one correspondence from a family of $n-1$ dimensional subspaces to the line, but there are infinite points on each $n-1$ dimensional subspace, thus the conclusion that there is a oneone correspondence between n-dimensional space and the line, and the points on n-dimensional space are as many that as on the line is obviously mistaken.

We correct Cantor' conclusion as the followings:

There is a one-one correspondence between any interval and the line, the points on any interval are as many as on the line. There is a one-one correspondence between any curve and the line, the points on any curve are as many as on the line. There is a one-one correspondence from a family of curves that cover completely the plane to the line; the curves are as many as pints on the line. There is a one-one correspondence from a family of surfaces which fill completely the 3-dimensional space to the line; the surfaces in 3-dimensional space are as many as the points on the line. There is a one-one correspondence from a family of $n-1$ dimensional subspaces which fill in the n-dimensional space to the points on the line. There is no one-one correspondence from the points in n-dimensional space to the points on the line.

Let . express the cardinality of a set, then

$$
C=\overline{\bar{E}}<\overline{\overline{E^{2}}}<\overline{\overline{E^{3}}}<\cdots<\overline{\overline{E^{n}}}<\cdots .
$$

In this paper, by using an appropriate monotone and continuous function, the one-one correspondence can be established between any two intervals; by using geometrical method or algebraic means, the one-one relation is established between the points on the curve and on the line; and by helping a appropriate continuous function of $\mathrm{n}$ variables, the correspondence from a family of $\mathrm{n}-1$ dimensional subspace in $n$-dimensional space to an interval. Thus, the paper can not only strengthen the relations among middle school maths, calculus, space analytic geometry, real function theory, can but also reduce the teaching difficulty of real analysis in some degree. After proposing the concept of moving curve (surface) and the concept of family curves (surfaces), and revealing the relations between moving curve (surface) and multivariate function, we can give the geometric meanings and the physics meanings of the problem of conditional extreme value. A new approach which is superior to current Lagrange multiplier method is obtained can completely replace it. Based on the concepts of moving curve (surface) and set theory, the method of changing directly multiple integrals or fist type surface integrals into simple integrals can have visual geometric meanings and physics meanings, and it is absolutely necessary to redefine multiple function integrals.

Thank Library of Chengdu Normal University!

\section{References}

[1] Ling, L.J. (2007) Selected Mathematical History (Optional Courses 3-1). The People’s Education Press, Beijin, 71-76.

[2] Zhang, H. (2012) Brief History of Mathematics. Science Press, Beijin, 227.

[3] Cheng, C.D. (2012) Introduction of Real Function. Science Press, Beijin, 17.

[4] Zhou, X.W. and Sun, W.C. (2014) Real Function. Science Press, Beijin, 12.

[5] Tang, Y.Z. (2007) A Method of Changing Multiple Intefrals and Surface Integrals Directly into Simple Integrals. Studies in college Mathematics, 10, 10.

[6] Li, G.Q. and Li, S.Z. (2009) Mathematics (Volume One). Higher Education Press, Beijin, 19.

[7] Xiong, M. and Xiong, J. (2012) Infiltrating the thought of Limit in Teaching Real Number. Course Education Research, 1, 64 .

[8] Xiong, M. (2009) Construction of the Regional Elements of Differential Calculus and Re-integration into a Single-integral Directly. Journal of Sichuan College of Education, 25, 32.

[9] Xiong, M. (2010) Change Double Integral into Simple Integral with Area Elment. Studies in College Mathematics, 13, 115. 
[10] Xiong, M. (2010) Eight Propositions for Simplifing Calcuation of Multiintegrals. Journal of Southwest University for Nationalities (Natural Science Edition), 36, 595.

[11] Xiong, M. (2013) Moving Curve (Surface) and Degenerate Transformation for Multi-Integrals. Studies in College Mathematics, 16, 22. 